\title{
The Curvelet Approach for Denoising in various Imaging Modalities using Different Shrinkage Rules
}

\author{
D. Mary sugantharathnam \\ Assistant Professor \\ Government College of Engineering \\ Tirunelveli, India.
}

\author{
Dr. D. Manimegalai \\ Professor \\ National Engineering College \\ Kovilpatti, India.
}

\begin{abstract}
The images usually bring different kinds of noise in the process of receiving, coding and transmission. In this paper the Curvelet transform is used for de-noising of image. Two digital implementations of the Curvelet transform (a multiscale transform) viz the Unequally Spaced Fast Fourier Transform (USFFT) and the Wrapping Algorithm are used to de-noise images degraded by different types of noises such as Random, Gaussian, Salt and Pepper, Speckle and Poisson noise. This paper aims at the effect the Curvelet transform has in Curvelet shrinkage assuming different types of noise models. A signal to noise ratio as a measure of the quality of de-noising was preferred. The experimental results show that the conventional Curvelet shrinkage approach fails to remove Poisson noise in medical images.
\end{abstract}

\section{Key words}

Curvelet transform, Wrapping Transform, USFFT.

\section{INTRODUCTION}

The need for image enhancement and restoration is encountered in many practical applications. For efficient image representation, we should have multi-resolution, localization, directionality, critical sampling and anisotropy [5]. In practice, any image may be degraded by various types and forms of noise and the most common one is the additive noise.

In the field of scientific computing, wavelet and other related multi-scale methods give high speed computations [14]. Wavelets are found to be less effective for singularities along the lines. Research was then focused on providing better alternatives beyond wavelets by combining the ideas from geometry and multi-scale analysis. The Curvelet transform described in [10] is an efficient directional multi-resolution transform.

Curvelets form an effective model that not only considers a multiscale time frequency local partition but also uses geometric feature direction. This transform was designed to represent edges and other singularities along curves much more efficiently than traditional transforms (i.e.) using many fewer coefficients for a given accuracy of reconstruction.

The first generation Curvelet transform involves special partitioning of phase space followed by the Ridgelet Transform, which is applied to blocks of data that are well localized in space and frequency. These constructions of Curvelet transform is redundant and hence slow [2].
This paper aims at the analysis of de-noising of gray scale images using second generation Curvelet transform [4,14] i.e. transformation based on Unequally Spaced Fast Fourier transforms(USFFT) and transformation based on Wrapping [6] of specially selected Fourier samples which are faster and less redundant.

Curvelet threshold shrinkage algorithms are widely used in image de-noising. The principle is as follows: the large curvelet coefficients are viewed as actual image signal, while the small curvelet coefficients are viewed as noise signal. The thresholds are determined to a large extent by noise standard variance.

In this paper Curvelet de-noising techniques is applied to Natural images, Satellite images and Medical images such as Computed Tomography (CT) [7] \& Magnetic Resonance Imaging (MRI). A comprehensive evaluation of the Curvelet transform and the effect of Curvelet shrinkage techniques like Soft thresholding, Hard thresholding and Garrote thresholding as well as Partial reconstruction of curvelet coefficients for images corrupted with various types of noises like Random noise, Gaussian noise, Salt \& Pepper, Speckle and Poisson noise are presented in this paper. The focus is on the Donoho-Johnstone thresholding model to Curvelet transform applied to different imaging modalities corrupted by various types of noise[1].

Medical imaging system is very complex and often noisy owing to the physical mechanisms of the acquisition process. Most popular medical imaging modalities are degraded by some type of non Gaussian noise [9].

The literature is rich in de-noising methods assuming additive white Gaussian noise, but some of the important medical imaging modalities like CT, MRI, PET(Positron Emission Tomography) and SPECT(Single Photon Emission Computed Tomography) are corrupted by Poisson noise. Prior to effectively designing algorithms specifically for the removal of Poisson noise [3], it is essential to analyze the existing state of art method (i.e.) Curvelet thresholding / shrinkage and its efficiency in removal of Poisson noise in medical images [12].

The rest of the paper is organized as follows. In Section 2, the various Noise models are illustrated. The second generation Curvelet transform is discussed in Section 3 and the de-noising algorithm is described in Section 4. The experimental results are discussed in Section 5. Finally the conclusions are drawn in Section 6. The future work in removing Poisson noise in medical images is highlighted in section 7 . 


\section{NOISE MODELS}

Image noise is the random variation of brightness or color information in images produced by the sensor and circuitry of a scanner or digital camera. Image noise is generally regarded as an undesirable by product of image capture because it carries distortions present in the image that can obscure the subject of the photograph.

\subsection{Random noise}

It is a form of random stochastic process, characterized by large number of overlapping transient disturbances occurring at random, such as thermal noise and shot noise. Random noise is characterized by intensity and color fluctuations above and below the actual image intensity. The pattern of random noise changes even if the exposure settings are identical.

\subsection{Gaussian noise}

The standard model of amplifier noise is additive, Gaussian, independent at each pixel and independent of the signal intensity. Additive white Gaussian noise is a channel model in which the only impairment to communication is a linear addition of wideband or white noise with a constant spectral density and a Gaussian distribution of amplitude. Wideband Gaussian noise comes from many natural sources such as thermal vibrations of atoms in conductors, shot noise, black body radiation from the earth and other warm objects. This channel is a good model for many satellite and deep space communication links.

\subsection{Salt and Pepper Noise}

It represents itself as randomly occurring white and black pixels. Salt and pepper noise creeps into images in situations where quick transients, such as faulty switching take place.

\subsection{Speckle noise}

It is a granular noise that inherently exists in and degrades the quality of the active radar and Synthetic Aperture Radar images. It is a multiplicative noise that is; it is in direct proportion to local gray level in any area. The signal and the noise are statistically independent of each other. The sample mean and variance of a single pixel are equal to the mean and variance of the local area that is centred on that pixel.

\subsection{Poisson Noise}

Many images such as those from radiography, contains noise that satisfies a poisson distribution. The magnitude of Poisson noise varies across the image, as it depends on the image intensity. This makes removing such noise very difficult. Poisson images occur in many situations where image acquisition is performed using the detection of particles (e.g) counting photons being emitted from a radioactive source is applied in medical imaging like PET and SPECT and hence Poisson noise reduction is an essential problem.

Poisson noise is generated from the data instead of adding artificial noise to the data. For example if a pixel in an unsigned integer input has the value 10, then the corresponding output pixel will be generated from a Poisson distribution with a mean 10 .

\section{THE CURVELET TRANSFORM}

The Curvelet transform is a higher dimensional generalization of the wavelet transform designed to represent images at different scales and different angles. Curvelet transform is a special member of the multi-scale geometric transforms. It is a transform with multi-scale pyramid with many directions at each length scale. Curvelets will be superior over wavelets in following cases: [14]

- Optimally sparse representation of objects with edges

- Optimal image reconstruction in severely ill-posed problems

- Optimal sparse representation of wave propagators

The idea of the Curvelet transform is first to decompose the image into subbands, i.e. to separate the object into a series of disjoint scales. Curvelets are initially introduced by Candes and Donoho. The Discrete Curvelet transform (DCT) takes as input a Cartesian grid of the form $f\left(n_{1}, n_{2}\right), 0 \leq n_{1}, n_{2}<n$, and outputs a collection of coefficients $\mathrm{c}^{\mathrm{D}}(\mathrm{j}, 1, \mathrm{k})$ defined by

$$
C^{D}(j, k, l)=\sum_{n_{1}, n_{2}} f\left(n_{1}, n_{2}\right) \overline{\emptyset_{1, l, k}^{D}\left(n_{1}, n_{2}\right)}
$$

where $\emptyset_{\mathrm{j}, \mathrm{l}, \mathrm{k}}^{\mathrm{D}}\left(\mathrm{n}_{1}, \mathrm{n}_{2}\right)$ are digital curvelet waveforms which preserve the listed properties of the continuous curvelet. DCT can be implemented in two ways. The first method is based on unequally-spaced fast Fourier transform (USFFT) and the second is based on the Wrapping of specially selected Fourier samples [8]. The two implementations essentially differ by spatial grid used to translate curvelets at each scale and angle.

For the 2D image, the architecture of the DCT via Wrapping is as follows:

- $\quad$ Apply the 2D FFT and obtain Fourier samples

$$
\hat{\mathrm{f}}\left[\mathrm{n}_{1}, \mathrm{n}_{2}\right],-\mathrm{n} / 2 \leq \mathrm{n}_{1}, \mathrm{n}_{2}<n / 2
$$

- $\quad$ For each scale $j$ and angle $l$, form the product

$$
\widetilde{\mathrm{U}_{\mathrm{j}, 1}}\left[\mathrm{n}_{1}, \mathrm{n}_{2}\right] \hat{\mathrm{f}}\left[\mathrm{n}_{1}, \mathrm{n}_{2}\right]
$$

- Wrap this product around the origin and obtain

$$
\widetilde{\mathrm{f}_{\mathrm{j}, \mathrm{l}}}\left[\mathrm{n}_{1}, \mathrm{n}_{2}\right]=\mathrm{W}\left(\widetilde{\mathrm{U}_{\mathrm{j}, 1}} \hat{\mathrm{f}}\right)\left[\mathrm{n}_{1}, \mathrm{n}_{2}\right]
$$

- Apply the inverse $2 \mathrm{D}$ FFT to each $\widetilde{f_{J, l}}\left[n_{1}, n_{2}\right]$ hence collecting the discrete coefficients $c^{D}(j, l, k)$.

The curvelet denoising method consists of the following steps:

- Estimate the noise standard deviation $\sigma$ in the input image

- Calculate the Curvelet transform of the input image. We get a set of bands $w_{j}$, each band $w_{j}$ contains $\mathrm{N}_{\mathrm{j}}$ coefficients and corresponds to a given resolution level.

- Calculate the noise standard deviation $\sigma_{j}$ for each band $j$ of the Curvelet transform.

- For each band $j$ do : Calculate the maximum of the band and multiply each curvelet coefficient.

- Reconstruct the image from the modified curvelet coefficients. 


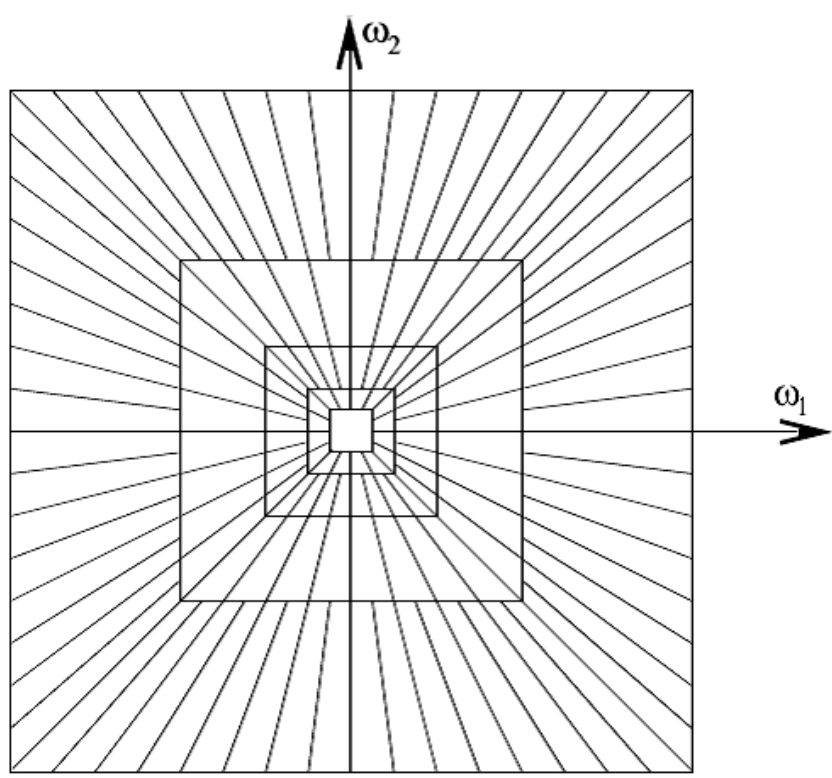

Fig.1 Curvelet tiling, also called second dyadic decomposition of the frequency plane.

\section{IMAGE DENOISING}

Image De-noising is used to produce good estimates of the original image from noisy observations. The restored image should contain less noise than the observations while still keep sharp transitions (i.e edges).

Suppose an image $f(m, n)$ is corrupted by the additive noise [15] $\mathrm{g}(\mathrm{m}, \mathrm{n})=\mathrm{f}(\mathrm{m}, \mathrm{n})+\eta(\mathrm{m}, \mathrm{n})$

where $\eta(m, n)$ are independent identically distributed Gaussian random variable with zero mean and variance $\sigma^{2}$.

Image de-noising algorithms vary from simple thresholding to complicate model based methods. However simple thresholding methods can remove most of the noise.

\subsection{Algorithm}

1. Apply the Forward Curvelet transform to the noisy image.

2. Threshold the Curvelet co-efficients to remove some insignificant curvelet co-efficients by using a thresholding function in the curvelet domain.

3. Inverse Curvelet transform of the thresholded co-efficients to reconstruct a function.

\subsection{Curvelet Shrinkage/Thresholding}

Shrinkage/thresholding plays an important role in curvelet application. Various thresholding techniques have been applied on the curvelet co-efficients of the observed image. The small co-efficients are dominated by noise, while co-efficients with large absolute value carry more signal information than noise. As a result noisy co-efficients (small co-efficients below a certain threshold value) are replaced by zero.

The curvelet shrinkage is taken as

$$
\mathrm{P}_{\sigma} \mathrm{u}=\mathrm{T}^{-1} \mathrm{~S}_{\sigma} \mathrm{T}(\mathrm{u})
$$

$\mathrm{T}$ denotes the Curvelet transform, $\mathrm{T}^{-1}$ the inverse transform and $\mathrm{S}_{\sigma}$ is the thresholding function.

\subsection{Thresholding Function[10]}

1. Soft Thresholding is defined by a fixed threshold $\sigma>0$

$$
S_{\sigma}(x)=\left\{\begin{array}{lc}
x-\sigma & x \geq \sigma \\
0 & |x|<\sigma \\
x+\sigma & x \leq \sigma
\end{array}\right.
$$

2. Hard Thresholding

3. Continuous Garrote thresholding

$$
\mathrm{S}_{\sigma}(\mathrm{x})= \begin{cases}\mathrm{x} & |\mathrm{x}| \geq \sigma \\ 0 & |\mathrm{x}|<\sigma\end{cases}
$$

$$
S_{\sigma}(x)= \begin{cases}x-\frac{\sigma^{2}}{x} & |x| \geq \sigma \\ 0 & |x|<\sigma\end{cases}
$$

These thresholding functions might be a good choice because large co-efficients remain nearly unaltered. In partial reconstruction the image was reconstructed using the few largest co-efficients and the remaining co-efficients were set to zero.

\section{RESULTS AND DISCUSSION}

The experiments are conducted on several types of gray scale images of size 256 x 256 in MATLAB platform and the Curvelet transform via USFFT and Wrapping technique was implemented based on curvelet software package [10].

CT scan images and MRI scan images of brain slice, Natural images and Satellite images are de-noised using Curvelet transform via USFFT and Wrapping Technique. Various types of noise namely Random noise, Additive white Gaussian noise, Speckle noise, Salt and Pepper and Poisson noise are added.

The effectiveness of the Curvelet shrinkage methods as well as partial reconstruction of Curvelet coefficients are demonstrated for various gray scale images with a noise factor of $\sigma=20$ for Random noise, noise density of 0.05 for Salt \& Pepper noise, a multiplicative noise factor of 0.04 for Speckle noise and for Gaussian white noise a mean of 0 and a variance of 0.01 is used.

To compare the results of different curvelet thresholding techniques, a image similarity measure is used.

$$
\text { PSNR }=10 \log _{10}\left(\frac{\mathrm{f}_{\mathrm{max}}^{2}}{\mathrm{MSE}}\right)
$$

where fmax is the maximum value of the image intensities and MSE is the mean square error between the reconstructed image and original one

$$
\mathrm{MSE}=\frac{1}{\mathrm{MN}} \sum_{\mathrm{m}=0}^{\mathrm{M}-1} \sum_{\mathrm{n}=0}^{\mathrm{N}-1}|\mathrm{f}(\mathrm{m}, \mathrm{n})-\tilde{\mathrm{f}}(\mathrm{m}, \mathrm{n})|^{2}
$$

where $f(m, n)$ is the original image and $\tilde{f}(m, n)$ is the de-noised image. $\mathrm{M} \times \mathrm{N}$ is the number of pixels. The de-noised image is closer to the original one when PSNR is higher.

The average PSNR measurements of various gray scale images degraded by Random noise, Salt \& Pepper noise, Speckle noise, Gaussian noise and Poisson noise with the two digital implementations of Curvelet transform are listed in tables 1 to 8 .

The denoising results for various gray scale images corrupted with Random noise, Salt and Pepper noise, Gaussian noise, Speckle and Poisson noise are shown in figures 2 to 6 . The application of 
Curvelet transform in image de-noising has been reported in [2], which demonstrated the advantage of curvelet over wavelet based image de-noising algorithm in terms of PSNR. In this experiment curvelet based image de-noising based on different curvelet threshold shrinkage rules applied to several gray scale imaging modalities is compared and the suitability of a curvelet thresholding technique in the removal of particular noise for a specific imaging modality is evaluated in terms of PSNR.

\subsection{Comparison on natural images:}

Experiments were conducted on 3 test images 'Lena', 'Barbara' and 'pout' of size $256 \times 256$ pixels. The result shows that the Curvelet transform implemented with USFFT and Wrapping technique using Hard and Garrote thresholding have strong capability of de-noising images degraded by Random noise, Salt \& Pepper noise, Speckle noise and Poisson noise. The results are listed in tables 1 and 5. However it is observed that soft thresholding is effective in removing Salt \& Pepper noise as compared to other thresholding techniques.

\subsection{Comparison on Medical Images:}

The experiment was simulated on 3 test images each; CT \& MRI of size $256 \times 256$ pixels. The results depicts that Hard \& Garrote thresholding of curvelet coefficients using USFFT and Wrapping Algorithms are efficient in the removal of Random noise, Gaussian noise \& Speckle noise in biomedical images. However the efficiency in the removal of Salt \& Pepper noise is due to soft thresholding of curvelet coefficients. The results are tabulated in tables 2, 3, 6 and 7 .

\subsection{Comparison on satellite images:}

The Curvelet transform was also tested on satellite images of size $256 \times 256$ pixels. The result obtained indicate the similarity in the performance of curvelet thresholding to that of natural images, viz., Hard \& Garrote thresholding being efficient in de-noising images degraded by Random noise, Gaussian noise, Speckle \& Poisson noise. Table 4 and 8 illustrates the performance of curvelet denoising on satellite images.

Soft thresholding of curvelet coefficients delivers better results compared with the other thresholding techniques in the removal of Salt and Pepper noise. It is also observed that all curvelet thresholding techniques presented in this paper fails to remove Poisson noise in medical images. The results are shown in tables 2, 3, 6 and 7 .

The best performance of the Curvelet transform via USFFT and Wrapping techniques implemented with curvelet thresholding and partial reconstruction in de-noising various types of noise in terms of average PSNR gain [13] are illustrated in tables 9 to 13 .

i. The highest PSNR gain of 11.0dB approximately is obtained with Hard \& Garrote thresholding for Satellite image degraded by Random noise.

ii. Satellite and Natural images degraded by Salt \& Pepper noise achieves a highest PSNR gain of $6.3 \mathrm{~dB}$ using Soft thresholding technique.

iii. Hard \& Garrote thresholding delivers better results for Natural images degraded by Gaussian noise, the highest PSNR gain being $8.3 \mathrm{~dB}$ for Curvelet transform implemented with USFFT and $8.7 \mathrm{~dB}$ for Wrapping technique.

iv. Garrote thresholding offers the highest PSNR gain of $8.5 \mathrm{~dB}$ for natural images corrupted by Speckle noise.

v. Poission noise to some extent is being removed in Natural images using Hard \& Garrote thresholding. Table 13 clearly depicts a negative gain for almost all the imaging modalities implemented with the four de-noising techniques in the removal of Poisson noise.

\section{CONCLUSIONS}

In this paper a strategy for digitally implementing the Curvelet transform via USSFT and Wrapping technique is presented. The Curvelet transform is capable of resolving 2D singularities and represents edges more efficiently in images. The resulting implementations have the exact reconstruction property and give stable reconstruction.

The experimental results showed that the Curvelet transform implemented with USFFT \& Wrapping Algorithm using Hard \& Garrote thresholding consistently produces the highest PSNR for Bio medical images, satellite images \& natural images degraded by Random noise, Gaussian noise and Speckle noise, while soft thresholding offers best results for de-noising Salt \& Pepper noise for all the test images used.

Though best results are obtained while de-noising all the test images corrupted by Random noise, Gaussian noise, Speckle noise, and Salt and Pepper noise in terms of PSNR gain it is noticed that lowest PSNR gain is obtained for Bio-medical images when compared to satellite \& Natural images.

The experimental results also clearly indicates that Curvelet transform using thresholding techniques proves to be inferior in de-noising Bio-medical images corrupted by Random noise, Gaussian noise, Speckle noise and Salt and Pepper noise.

Curvelet thresholding techniques proves to be a failure in the removal of Poisson noise in Bio-medical images, a factor noteworthy to be mentioned in the paper.

Partial reconstruction of curvelet coefficients proves to be a failure for all types of noises tested with various images. However the visual quality of the images is preserved to a certain extent.

Both the transforms have low running times, but however USFFT is slower than Wrapping algorithm. The Computation time of the tested methods using Matlab codes on a $2.19 \mathrm{GHz}$ PC gives 5.6 seconds for the Curvelet transform implemented with USFFT and 0.2 seconds for Wrapping technique.

\section{FUTURE WORK}

Future research will focus on finding a novel method for removing Poisson noise in medical images, especially in PET (Positron Emission Tomography) data which uses Multiscale Variance stabilizing transforms (MS-VST) [11] which combines the VST with the low pass filters involved in various multiscale multidirection transforms (MS-MD). 
Table1: De-noising results (PSNR in $\mathrm{dB}$ ) with curve-let via USFFT for the four de-noising methods for natural images

\begin{tabular}{|l|c|c|c|c|c|}
\hline NOISE & $\begin{array}{c}\text { NOISY } \\
\text { IMAGE }\end{array}$ & SOFT & HARD & GARROTE & $\begin{array}{c}\text { PARTIAL } \\
\text { RECONSTRUCTION }\end{array}$ \\
\hline Random & 22.0800 & 27.3700 & $\mathbf{2 9 . 7 6 0 0}$ & $\mathbf{2 9 . 7 7 0 0}$ & 24.2600 \\
\hline $\begin{array}{l}\text { Salt \& } \\
\text { Pepper }\end{array}$ & 18.5200 & $\mathbf{2 4 . 8 0 0 0}$ & 20.8400 & 20.9200 & 19.7200 \\
\hline Gaussian & 19.9700 & 27.0100 & $\mathbf{2 8 . 2 4 0 0}$ & $\mathbf{2 8 . 2 4 0 0}$ & 22.3900 \\
\hline Speckle & 20.3000 & 27.2200 & $\mathbf{2 7 . 7 4 0 0}$ & $\mathbf{2 8 . 7 9 0 0}$ & 22.6800 \\
\hline Poisson & 27.3800 & 27.2700 & $\mathbf{3 0 . 1 8 0 0}$ & $\mathbf{3 0 . 1 7 0 0}$ & 27.9900 \\
\hline
\end{tabular}

Table2: De-noising results (PSNR in $\mathrm{dB}$ ) with curve-let via USFFT for the four de-noising methods for CT images

\begin{tabular}{|l|c|c|c|c|c|}
\hline NOISE & $\begin{array}{c}\text { NOISY } \\
\text { IMAGE }\end{array}$ & SOFT & HARD & GARROTE & $\begin{array}{c}\text { PARTIAL } \\
\text { RECONSTRUCTION }\end{array}$ \\
\hline Random & 22.0670 & 25.7900 & 29.2800 & 29.2900 & 24.0100 \\
\hline $\begin{array}{l}\text { Salt \& } \\
\text { Pepper }\end{array}$ & 17.0800 & $\mathbf{2 1 . 9 0 0 0}$ & 19.1000 & 19.1000 & 18.3100 \\
\hline Gaussian & 20.9800 & 24.2000 & $\mathbf{2 6 . 6 1 0 0}$ & $\mathbf{2 6 . 5 7 0 0}$ & 22.5600 \\
\hline Speckle & 22.4800 & 24.6800 & $\mathbf{2 7 . 6 1 0 0}$ & $\mathbf{2 7 . 6 5 0 0}$ & 23.2100 \\
\hline Poisson & 29.8000 & 25.5900 & $\mathbf{2 9 . 8 0 0 0}$ & $\mathbf{2 9 . 8 1 0 0}$ & 27.5000 \\
\hline
\end{tabular}

Table3: De-noising results (PSNR in $\mathrm{dB}$ ) with curve-let via USFFT for the four de-noising methods for MRI images

\begin{tabular}{|l|c|c|c|c|c|}
\hline NOISE & $\begin{array}{c}\text { NOISY } \\
\text { IMAGE }\end{array}$ & SOFT & HARD & GARROTE & $\begin{array}{c}\text { PARTIAL } \\
\text { RECONSTRUCTION }\end{array}$ \\
\hline Random & 22.0900 & 26.2700 & $\mathbf{2 8 . 3 3 0 0}$ & $\mathbf{2 8 . 4 0 0 0}$ & 24.5000 \\
\hline $\begin{array}{l}\text { Salt \& } \\
\text { Pepper }\end{array}$ & 16.4500 & $\mathbf{2 1 . 5 0 0 0}$ & 18.2000 & 18.4000 & 17.6600 \\
\hline Gaussian & 21.5900 & 24.2000 & $\mathbf{2 5 . 5 0 0 0}$ & $\mathbf{2 5 . 5 0 0 0}$ & 23.2300 \\
\hline Speckle & 28.5000 & 26.1000 & $\mathbf{2 8 . 3 9 0 0}$ & $\mathbf{2 8 . 3 3 0 0}$ & 27.4800 \\
\hline Poisson & 34.4500 & 26.2000 & 28.6600 & 28.6300 & $\mathbf{3 0 . 4 4 0 0}$ \\
\hline
\end{tabular}

Table4: De-noising results (PSNR in $\mathrm{dB}$ ) with curve-let via USFFT for the four de-noising methods for satellite images

\begin{tabular}{|l|c|c|c|c|c|}
\hline NOISE & $\begin{array}{c}\text { NOISY } \\
\text { IMAGE }\end{array}$ & SOFT & HARD & GARROTE & $\begin{array}{c}\text { PARTIAL } \\
\text { RECONSTRUCTION }\end{array}$ \\
\hline Random & 22.0400 & 30.7300 & $\mathbf{3 3 . 1 0 0 0}$ & $\mathbf{3 2 . 9 9 0 0}$ & 23.1100 \\
\hline $\begin{array}{l}\text { Salt \& } \\
\text { Pepper }\end{array}$ & 16.7600 & $\mathbf{2 3 . 0 3 0 0}$ & 19.0500 & 19.0500 & 18.1000 \\
\hline Gaussian & 21.1800 & 26.5000 & $\mathbf{2 7 . 3 0 0 0}$ & $\mathbf{2 7 . 2 7 0 0}$ & 23.2000 \\
\hline Speckle & 25.7100 & 30.7200 & $\mathbf{3 3 . 1 2 0 0}$ & $\mathbf{3 3 . 1 0 0 0}$ & 26.7600 \\
\hline Poisson & 32.2900 & 30.7500 & $\mathbf{3 3 . 7 8 0 0}$ & $\mathbf{3 3 . 7 8 0 0}$ & 32.3100 \\
\hline
\end{tabular}

Table5: De-noising results (PSNR in dB) with curve-let via Wrapping for the four de-noising methods for natural images

\begin{tabular}{|l|c|c|c|c|c|}
\hline NOISE & $\begin{array}{c}\text { NOISY } \\
\text { IMAGE }\end{array}$ & SOFT & HARD & GARROTE & $\begin{array}{c}\text { PARTIAL } \\
\text { RECONSTRUCTION }\end{array}$ \\
\hline Random & 22.0700 & 28.0200 & $\mathbf{2 9 . 9 5 0 0}$ & $\mathbf{2 9 . 9 5 0 0}$ & 22.1200 \\
\hline $\begin{array}{l}\text { Salt \& } \\
\text { Pepper }\end{array}$ & 18.4100 & $\mathbf{2 4 . 7 2 0 0}$ & 20.8400 & 20.7500 & 18.5500 \\
\hline Gaussian & 19.9800 & 27.0400 & $\mathbf{2 8 . 7 0 0 0}$ & $\mathbf{2 8 . 4 1 0 0}$ & 20.0700 \\
\hline Speckle & 20.2700 & 27.2500 & $\mathbf{2 8 . 8 3 0 0}$ & $\mathbf{2 8 . 8 3 0 0}$ & 20.3100 \\
\hline Poisson & 26.9500 & 27.6400 & $\mathbf{3 0 . 3 3 0 0}$ & $\mathbf{3 0 . 2 7 0 0}$ & 27.4400 \\
\hline
\end{tabular}

Table6: De-noising results (PSNR in $\mathrm{dB}$ ) with curve-let via Wrapping for the four de-noising methods for CT images

\begin{tabular}{|l|c|c|c|c|c|}
\hline NOISE & $\begin{array}{c}\text { NOISY } \\
\text { IMAGE }\end{array}$ & SOFT & HARD & GARROTE & $\begin{array}{c}\text { PARTIAL } \\
\text { RECONSTRUCTION }\end{array}$ \\
\hline Random & 22.0500 & 26.5600 & $\mathbf{2 9 . 5 4 0 0}$ & $\mathbf{2 9 . 5 3 0 0}$ & 22.1200 \\
\hline $\begin{array}{l}\text { Salt \& } \\
\text { Pepper }\end{array}$ & 17.1700 & $\mathbf{2 2 . 0 8 0 0}$ & 19.1500 & 19.2100 & 17.2600 \\
\hline Gaussian & 20.9600 & 24.3000 & $\mathbf{2 7 . 1 5 0 0}$ & $\mathbf{2 6 . 6 6 0 0}$ & 21.1800 \\
\hline Speckle & 22.5100 & 24.8000 & $\mathbf{2 7 . 7 4 0 0}$ & $\mathbf{2 7 . 8 0 0 0}$ & 22.5200 \\
\hline Poisson & 29.8500 & 25.6800 & $\mathbf{3 0 . 1 0 0 0}$ & $\mathbf{3 0 . 1 0 0 0}$ & 29.9100 \\
\hline
\end{tabular}

Table7: De-noising results (PSNR in $\mathrm{dB}$ ) with curve-let via Wrapping for the four de-noising methods for MRI images

\begin{tabular}{|l|c|c|c|c|c|}
\hline NOISE & $\begin{array}{c}\text { NOISY } \\
\text { IMAGE }\end{array}$ & SOFT & HARD & GARROTE & $\begin{array}{c}\text { PARTIAL } \\
\text { RECONSTRUCTION }\end{array}$ \\
\hline Random & 22.0600 & 26.2600 & $\mathbf{2 8 . 5 0 0 0}$ & $\mathbf{2 8 . 2 1 0 0}$ & 22.1100 \\
\hline $\begin{array}{l}\text { Salt \& } \\
\text { Pepper }\end{array}$ & 16.4100 & $\mathbf{2 1 . 5 4 0 0}$ & 18.3900 & 18.4500 & 16.5300 \\
\hline Gaussian & 21.6000 & 24.2800 & $\mathbf{2 6 . 2 0 0 0}$ & $\mathbf{2 5 . 6 1 0 0}$ & 22.0900 \\
\hline Speckle & 28.5100 & 26.1200 & $\mathbf{2 8 . 4 9 0 0}$ & $\mathbf{2 8 . 5 5 0 0}$ & 28.5900 \\
\hline Poisson & 34.4900 & 26.2000 & 28.7700 & 28.7900 & $\mathbf{3 4 . 5 7 0 0}$ \\
\hline
\end{tabular}

Table8: De-noising results (PSNR in $\mathrm{dB}$ ) with curve-let via Wrapping for the four de-noising methods for satellite images

\begin{tabular}{|l|c|c|c|c|c|}
\hline NOISE & $\begin{array}{c}\text { NOISY } \\
\text { IMAGE }\end{array}$ & SOFT & HARD & GARROTE & $\begin{array}{c}\text { PARTIAL } \\
\text { RECONSTRUCTION }\end{array}$ \\
\hline Random & 22.0800 & 30.7500 & $\mathbf{3 3 . 3 0 0 0}$ & $\mathbf{3 3 . 3 0 0 0}$ & 22.1100 \\
\hline $\begin{array}{l}\text { Salt \& } \\
\text { Pepper }\end{array}$ & 16.8400 & $\mathbf{2 3 . 1 1 0 0}$ & 19.2400 & 19.1300 & 16.8600 \\
\hline Gaussian & 21.2000 & 26.5700 & $\mathbf{2 8 . 1 1 0 0}$ & $\mathbf{2 7 . 2 8 0 0}$ & 21.5900 \\
\hline Speckle & 25.7200 & 30.8400 & $\mathbf{3 3 . 3 8 0 0}$ & $\mathbf{3 3 . 3 0 0 0}$ & 25.7800 \\
\hline Poisson & 32.2400 & 30.8200 & $\mathbf{3 3 . 9 5 0 0}$ & $\mathbf{3 3 . 8 8 0 0}$ & 32.2600 \\
\hline
\end{tabular}

Table 9 Average PSNR gain (dB) of Curvelet transforms via USFFT and Wrapping technique with Random noise

\begin{tabular}{|l|c|c|c|c|c|c|c|c|}
\hline \multirow{2}{*}{ Images } & \multicolumn{5}{|c|}{ USFFT } & \multicolumn{4}{c|}{ Wrapping } \\
\cline { 2 - 9 } & Soft & Hard & Garrote & $\begin{array}{c}\text { Partial } \\
\text { reconstruction }\end{array}$ & Soft & Hard & Garrote & $\begin{array}{c}\text { Partial } \\
\text { reconstruction }\end{array}$ \\
\hline CT & 3.6 & 7.2 & 702 & 1.9 & 4.51 & 7.5 & 7.5 & 0.05 \\
\hline MRI & 4.2 & 6.24 & 6.3 & 2.4 & 4.2 & 6.44 & 6.2 & 0.05 \\
\hline Satellite & 8.7 & $\mathbf{1 1 . 1}$ & $\mathbf{1 0 . 9 5}$ & 1.1 & 8.7 & $\mathbf{1 1 . 2}$ & $\mathbf{1 1 . 2}$ & 0.03 \\
\hline Natural & 5.3 & 7.7 & 7.7 & 2.2 & 5.95 & 7.9 & 7.9 & 0.05 \\
\hline
\end{tabular}

Table 10 Average PSNR gain (dB) of Curvelet transforms via USFFT and Wrapping technique with Salt and Pepper noise

\begin{tabular}{|l|c|c|c|c|c|c|c|c|}
\hline \multirow{2}{*}{ Images } & \multicolumn{5}{|c|}{ USFFT } & \multicolumn{4}{c|}{ Wrapping } \\
\cline { 2 - 9 } & Soft & Hard & Garrote & $\begin{array}{c}\text { Partial } \\
\text { reconstruction }\end{array}$ & Soft & Hard & Garrote & $\begin{array}{c}\text { Partial } \\
\text { reconstruction }\end{array}$ \\
\hline CT & 4.8 & 2.02 & 2.02 & 1.23 & 4.9 & 1.98 & 2.04 & 0.09 \\
\hline MRI & 5.1 & 1.75 & 1.95 & 1.21 & 5.13 & 1.98 & 2.04 & 0.12 \\
\hline Satellite & $\mathbf{6 . 3}$ & 2.3 & 2.3 & 1.34 & $\mathbf{6 . 3}$ & 2.4 & 2.3 & 0.02 \\
\hline Natural & $\mathbf{6 . 3}$ & 2.32 & 2.4 & 1.2 & $\mathbf{6 . 3}$ & 2.4 & 2.34 & 0.14 \\
\hline
\end{tabular}


Table 11 Average PSNR gain (dB) of Curvelet transforms via USFFT and Wrapping technique with Gaussian noise

\begin{tabular}{|l|c|c|c|c|c|c|c|c|}
\hline \multirow{2}{*}{ Images } & \multicolumn{5}{|c|}{ USFFT } & \multicolumn{5}{c|}{ Wrapping } \\
\cline { 2 - 9 } & Soft & Hard & Garrote & $\begin{array}{c}\text { Partial } \\
\text { reconstruction }\end{array}$ & Soft & Hard & Garrote & $\begin{array}{c}\text { Partial } \\
\text { reconstruction }\end{array}$ \\
\hline CT & 3.2 & 5.6 & 5.6 & 1.6 & 3.3 & 6.2 & 5.72 & 0.22 \\
\hline MRI & 2.6 & 3.91 & 3.91 & 1.64 & 2.7 & 4.6 & 4.0 & 0.49 \\
\hline Satellite & 5.32 & 6.12 & 6.1 & 2.0 & 5.4 & 6.9 & 6.1 & 0.39 \\
\hline Natural & 7.04 & $\mathbf{8 . 3}$ & $\mathbf{8 . 3}$ & 2.42 & 7.02 & $\mathbf{8 . 7}$ & $\mathbf{8 . 7}$ & 0.09 \\
\hline
\end{tabular}

Table 12 Average PSNR gain ( $\mathrm{dB}$ ) of Curvelet transforms via USFFT and Wrapping technique with Speckle noise

\begin{tabular}{|l|c|c|c|c|c|c|c|c|}
\hline \multirow{3}{*}{ Images } & \multicolumn{5}{|c|}{ USFFT } & \multicolumn{4}{c|}{ Wrapping } \\
\cline { 2 - 9 } & Soft & Hard & Garrote & $\begin{array}{c}\text { Partial } \\
\text { reconstruction }\end{array}$ & Soft & Hard & Garrote & $\begin{array}{c}\text { Partial } \\
\text { reconstruction }\end{array}$ \\
\hline CT & 2.2 & 5.13 & 5.17 & 0.73 & 2.3 & 5.23 & 5.3 & 0.01 \\
\hline MRI & -2.4 & -0.11 & -0.17 & -1.02 & -2.4 & -0.02 & -0.04 & -0.08 \\
\hline Satellite & 5.01 & 7.41 & 7.4 & 1.1 & 5.12 & 7.7 & 7.6 & 0.06 \\
\hline Natural & 6.92 & 7.44 & $\mathbf{8 . 4 9}$ & 2.4 & 6.98 & $\mathbf{8 . 5 6}$ & $\mathbf{8 . 5 6}$ & 0.04 \\
\hline
\end{tabular}

Table 13 Average PSNR gain (dB) of Curvelet transforms via USFFT and Wrapping technique with Poisson noise

\begin{tabular}{|l|c|c|c|c|c|c|c|c|}
\hline \multirow{2}{*}{ Images } & \multicolumn{5}{|c|}{ USFFT } & \multicolumn{4}{c|}{ Wrapping } \\
\cline { 2 - 9 } & Soft & Hard & Garrote & $\begin{array}{c}\text { Partial } \\
\text { reconstruction }\end{array}$ & Soft & Hard & Garrote & $\begin{array}{c}\text { Partial } \\
\text { reconstruction }\end{array}$ \\
\hline CT & -4.2 & 0.0 & 0.01 & -2.3 & -4.2 & 0.25 & 0.25 & 0.06 \\
\hline MRI & -8.3 & -5.8 & -5.8 & -4.0 & -8.3 & -5.7 & -5.7 & 0.08 \\
\hline Satellite & -1.54 & 1.5 & 1.5 & 0.59 & -1.42 & $\mathbf{1 . 7 1}$ & $\mathbf{1 . 6 4}$ & 0.02 \\
\hline Natural & 0.11 & $\mathbf{2 . 8}$ & $\mathbf{2 . 8}$ & 0.6 & 0.69 & $\mathbf{3 . 3 8}$ & $\mathbf{3 . 3 2}$ & 0.49 \\
\hline
\end{tabular}

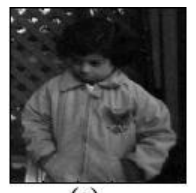

(a)

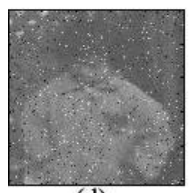

(d)

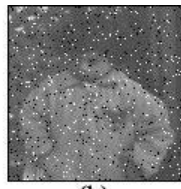

(b)

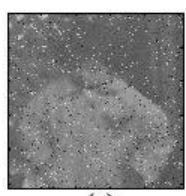

(e)

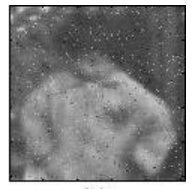

(c)

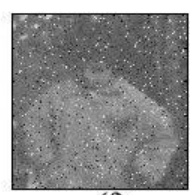

(f)
Figure 2:De-noising results for Natural image (Salt $\&$ Pepper) via USFFT (a) Original (b) Noisy (PSNR=18.7981) (c) Soft $(P S N R=27.4305)$ (d) Hard (PSNR=21.6223) (e) Garrote (PSNR=21.7859) (f) Partial Reconstruction $(P S N R=20.2025)$

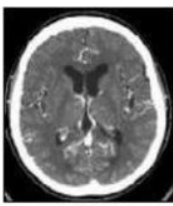

(a)

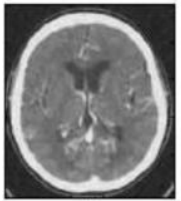

(d)

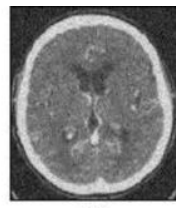

(b)

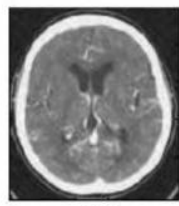

(e)

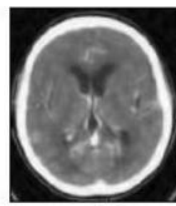

(c)

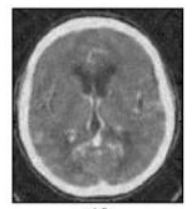

(f)
Figure 3: De-noising results for CT image (Random Noise) via USFFT (a) Original (b) Noisy (PSNR=22.0519) (c) Soft

(PSNR=26.4872) (d) Hard (PSNR=29.9437) (e) Garrote (PSNR=29.7930) (f) Partial Reconstruction (PSNR=24.4251)

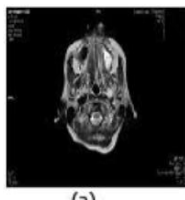

(a)

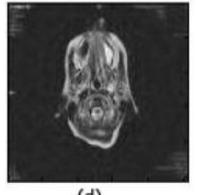

(d)

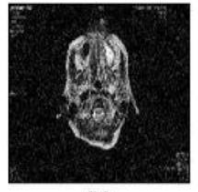

(b)

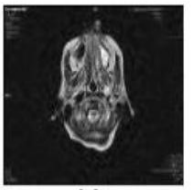

(e)

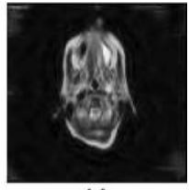

(c)

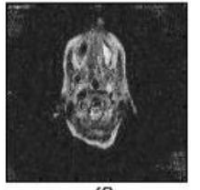

(f)
Figure 4:De-noising results for MRI image (Gaussia) via Wrapping (a) Original (b) Noisy (PSNR=21.6901) (c) Soft (PSNR=23.3243) (d) Hard (PSNR=25.2763) (e) Garrote (PSNR=24.7916) (f) Partial Reconstruction (PSNR=22.1776)

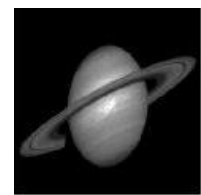

(a)

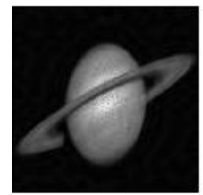

(d)

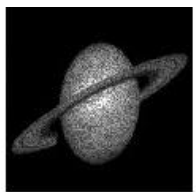

(b)

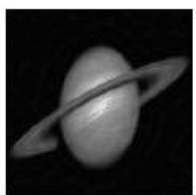

(e)

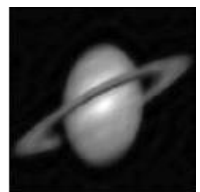

(c)

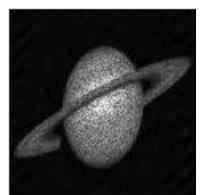

(f)
Figure 5:De-noising results for Satellite image (Speckle Noise) via USFFT (a) Original (b) Noisy (PSNR=26.8288) (c) Soft (PSNR=33.7775) (d) Hard (PSNR=36.6704) (e) Garrote (PSNR=36.4842) (f) Partial Reconstruction $(\mathrm{PSNR}=27.7541)$ 


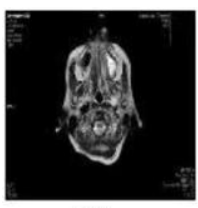

(a)

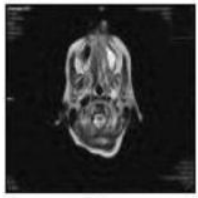

(d)

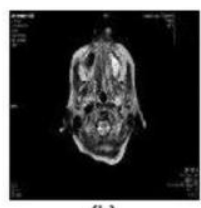

(b)

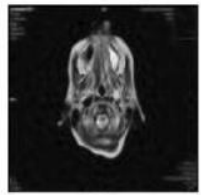

(d)

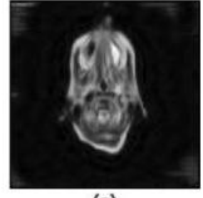

(c)

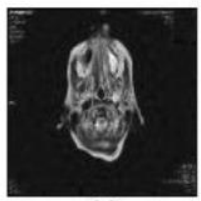

(e)
Figure 6:De-noising results for MRI image (Poisson Noise) via Wrapping (a) Original (b) Noisy (PSNR=34.6454) (c) Soft (PSNR=25.2912) (d) Hard (PSNR=28.0567) (e) Garrote (PSNR=28.0737) (f) Partial Reconstruction (PSNR=34.7231)

\section{ACKNOWLEDGEMENT}

First and foremost I would like to express my gratitude to the Almighty. It is honor for me to thank IJCA to share the significance of this paper. Our thanks to Mr. B. Ganapathy Ram , Adhoc Lecturer, Department of ECE, Government College of Engineering, Tirunelveli, Tamilnadu, who has contributed towards alignment of this manuscript.

\section{REFERENCES}

[1] D.L.Donoho. 1995. "De-noising by soft-thresholding", IEEE Transactions on Information Theory, Vol 41, No3, May 1995.

[2] Jean-Lue Starck, Emmanuel J. Candes and David L.Donoho. 2002. "The Curvelet transform for Image Denoising" IEEE Transactions on Image Processing, Vol 11, No 6, June 2002.

[3] Yeqiu Li, Jianming Lu, Ling Wang. 2005. "Removing Poisson Noise From Images In Wavelet Domain",2005 IEEE

[4] E.Candes \& D.L.Donoho,"Fast Discreet Curvelet transform", Stanford University, July2005.

[5] Latha Partheban \& R.Subramanian. 2006. "Medical Image Denoising using X-lets",2006 IEEE

[6] Prit Naik and shalini Bhatia. 2007. " Image De-noising using Curvelet transform", Proceedings of SPIT-IEEE colloquium and International conference, Mumbai, India, Vol.2007

[7] R.Sivakumar. 2007. " De-noising of Computer Tomography Images using Curvelet transform", ARPN Journal of Engineering and applied Sciences", Vol2, No1, February 2007.

[8] Jiang Tao, Zhao Xin. 2008. "Research and Application of Image Denoising Method Based on Curvelet Transform” The International Archives of the Photogrammetry, Remote Sensing and Spatial Information Sciences", Vol XXXVII Part B2, Beijing 2008.
[9] Isabel Rodrigues, Joao Sanches and Jose Bioucas-Dias. 2008. "Denoising of Medical Images corrupted by Poisson Noise" ICIP 2008: 1756-1759 ...

[10] Jianwei Ma and Gerlind Plonka. 2009. Computing with curvelets, From Image Processing to Turbulent Flows. 15219615/09/2009 IEEE, Computing in Science and Engineering,2009

[11] Arnaud De Decker, John Aldo Lee and Michel Verlysen. 2009."Variants Stabilizing Transformation In Patch-Based Bilateral Filters for Poisson Noise Image Denoising” 2009 IEEE

[12] Al-dahoudAli, Preeti D. Swami and J. Singhai. 2010. "Modified Curvelet thresholding for Image De-Noising", Journal of computer Science6(1): 18-23,2010.

[13] Jun Xu, Lei Yang, Dapeng Wu. 2010. " Ripplet : A new transform for image processing", J.Vis. commun.Image R. 21(2010) 627-639

[14] Ke Ding. 2010. "Wavelets, curvelets and Wave Atoms for Image Denoising", $20103^{\text {rd }}$ International Congress on Image \& Signal Processing(CISP 2010).

[15] RafaelC. Gonzalez and Richard E. Woods," Digital Image Processing"Second Edition

\section{AUTHORS PROFILE}

D. Mary sugantharathnam received her B.E(ECE) from Madurai Kamaraj University and M.E(Medical Electronics) from Anna University ,Chennai ,Tamilnadu ,India.. Currently she is working as Asst. Prof in ECE Department in Government College of Engineering, Tirunelveli. She has published several papers in National Conferences. Her area of interest are Digital image processing, Neural Networks and Soft Computing..

Dr.D.Manimegalai had her BE \& ME from Government College of Technology, Coimbatore and $\mathrm{PhD}$ from Manonmanium Sundaranar University, Tirunelveli. She worked in PSNA College of Engineering, Dindigul from 1985 - 1987. Since 1987, she has been working in National Engineering College in various positions. She has modest number of research publications including journals such as AMSE and Pattern Recognition letter and in National and International Conferences. She is also recognized supervisor for guiding $\mathrm{Ph} . \mathrm{D}$ students by various universities. Her Current area of research interests includes Medical Image Processing, Data Mining and Image Retrieval. She is a life member of Computer Society of India, System Society of India and Indian Society for Technical Education and a fellow member in Institution of Engineers. 